Research Article

\title{
Letrozole and the Traditional Chinese Medicine, Shaofu Zhuyu Decoction, Reduce Endometriotic Disease Progression in Rats: A Potential Role for Gut Microbiota
}

\author{
Ying Cao $\mathbb{D}^{\mathbb{D}},{ }^{1}$ Chunhua Jiang $\mathbb{D},{ }^{1}$ Yongsen Jia $\mathbb{D}^{\mathbb{C}},{ }^{1}$ Dingjie Xu $\mathbb{D}^{1},{ }^{1}$ and Yanyan $Y u \mathbb{D}^{2}$ \\ ${ }^{1}$ College of Traditional Chinese Medicine, North China University of Science and Technology, Tangshan, China \\ ${ }^{2}$ Reproductive Endocrinology Center, Dongfang Hospital, Beijing University of Chinese Medicine, Beijing, China \\ Correspondence should be addressed to Yanyan Yu; bjyuyanyan@163.com
}

Received 10 May 2020; Accepted 15 June 2020; Published 20 July 2020

Guest Editor: Jiangquan Liao

Copyright (c) 2020 Ying Cao et al. This is an open access article distributed under the Creative Commons Attribution License, which permits unrestricted use, distribution, and reproduction in any medium, provided the original work is properly cited.

\begin{abstract}
We previously showed that the Chinese herbal medicine, Shaofu Zhuyu decoction (SFZYD), shrank the size of endometriotic lesions in rats with endometriosis. We therefore conducted the present study to investigate the effects of letrozole and SFZYD on gut microbiota in endometriotic rats. Rats were divided into four groups: a blank group, model group, letrozole group, and SFZY group. Ectopic lesion size and COX-2 expression in the endometrium and endometriotic lesions were compared, and the community of gut microbiota was detected using $16 \mathrm{~S}$ rRNA gene sequencing. Both letrozole and SFZYD reduced the size of ectopic lesions as well as lowered the expression of COX-2, thus reducing the inflammatory response. Compared with the blank group, the $\alpha$-diversity of gut microbiota in endometriotic rats decreased, the Firmicutes/Bacteroidetes ratio increased, and the abundance of Ruminococcaceae was reduced. The $\alpha$-diversity of gut microbiota in the letrozole group was similar to that in the model group, but the Firmicutes/Bacteroidetes ratio was diminished. The $\alpha$-diversity in the SFZY group was similar to that in the blank group, the Firmicutes/Bacteroidetes ratio was attenuated, and the abundance of Ruminococcaceae was elevated compared with the model group. These results indicated that the therapeutic mechanisms of both letrozole and SFZYD were related to the restoration of gut microbiota.
\end{abstract}

\section{Introduction}

Endometriosis (EMs) is an estrogen-dependent disease in which endometrial glands and stromal tissues are implanted outside the uterine cavity. The incidence of EMs is $10 \%$ in women of childbearing age and up to $30-45 \%$ in infertile women [1]. The primary manifestations include dysmenorrhea, chronic pelvic pain, and infertility that seriously affect the quality of life of patients. Although there are many etiologic theories regarding EMs, they cannot adequately explain the occurrence and development of the disease. Moreover, its arcane etiology is an important reason hindering research on treatment for EMs.

According to the ectopic implantation theory accepted by most scholars, EMs originated from the shedding of endometrial debris that then enters the pelvic cavity with countercurrent menstrual blood flow [2]. The immune cells in the pelvic cavity are presumed to eliminate these cells, but the endometrial cells survive and lead to a continuous inflammatory status in the pelvic cavity due to the abnormal immunity in this region $[3,4]$. In fact, EMs is believed to be a chronic inflammatory disease from an increasing number of studies [5, 6]. Investigators have demonstrated an increase in the number of activated macrophages and proinflammatory cytokines and angiogenic factors in the pelvic fluid of EMs patients $[7,8]$, which could provide a favorable environment for ectopic endometrial implantation [8]. Compared with normal pelvic fluid, the pelvic fluid in EMs patients promotes the expression of the vascular endothelial growth factor (VEGF) and urokinase plasminogen activator (uPA) in endometrial cells [9]. Lipopolysaccharide (LPS), a common endotoxin, is at a much higher concentration in the 
menstrual blood of EMs patients relative to women without EMs, and the combination of LPS and toll-like receptor 4 (TLR4) can promote the proliferation of eutopic endometrial stromal cells. The quantity of $E$. coli (i.e., the principal source of LPS in the menstrual blood of EMs patients) is also much higher than that in the normal population [10].

The gut microbiota is known to be intimately involved in inflammatory responses. Karmarkar and Rock found that gut microbiota activated neutrophils via the myeloid differentiation primary response 88 (MyD88) pathway, which was a prerequisite for a pelvic inflammatory response [11]. Emani et al. found that abnormal gut microbiota weakened the function of the intestinal barrier, thereby leading to the leakage of bacteria into the pelvic cavity [12], and simultaneously facilitated the translocation of LPS from the intestinal epithelium to the pelvic cavity [13]. In addition, gut microbiota can affect the estrogen concentrations in circulation [14]. Therefore, researchers have increasingly focused on the relationship between the gut microbiota and the progression of EMs. Previous studies have confirmed that a mouse model of EMs exhibits dysbiosis of the gut microbiota [15]. Wide-spectrum antibiotics (e.g., vancomycin, neomycin, metronidazole, and ampicillin) inhibited the growth of ectopic lesions in EMs mice, and the oral gavage of feces from mice with EMs restored the growth of endometriotic lesions and inflammation in metronidazoletreated mice [16].

Letrozole, as a third-generation aromatase inhibitor, can inhibit the production of circulating and local estrogen and has been used in the experimental treatment of EMs in animal and clinical studies [17]. Shaofu Zhuyu decoction (SFZYD) is a classic prescription of the traditional Chinese medicine commonly used in treating dysmenorrhea; it originated from the Correction of the Errors of Medical Works by Wang Qingren, a physician in the Qing Dynasty. SFZYD is now widely used in TCM therapy for endometriosis. Our previous study showed that SFZYD inhibited cellular proliferation, promoted apoptosis, and reduced angiogenesis in ectopic endometrial tissues, thus playing a role in the treatment of endometriosis [18]. The purpose of the present study is to investigate our hypothesis that letrozole and SFZYD act on gut microbiota to inhibit the progression of lesions in rats with EMs.

\section{Materials and Methods}

2.1. Chemicals and Reagents. Estradiol valerate (Delpharm Lille S.A.S., France) and letrozole (Hengrui Medicine, China) were purchased from the eye hospital of the China Academy of Chinese Medical Sciences. Pentobarbital sodium and rabbit polyclonal antibody against cyclooxygenase-2 (ab15191; Abcam, UK) were purchased from Beijing Diweile Biotechnology Co., Ltd. The SFZYD contained 11 herbs: Foeniculi Fructus, Zingiberis Rhizoma, Corydalis Rhizoma, Myrrha, Rhizoma, Angelica Sinensis Radix, Radix Paeoniae Rubra, Cortex Cinnamomi, Typhae Pollen, and Trogopteri Feces. The origins, medicinal parts, and weight ratios of these herbs have been described in a previous article [18]. We created the formulation as follows.
In brief, all herbs except Cortex Cinnamomi were soaked in double-distilled water for $1 \mathrm{~h}$ and then decocted for $1 \mathrm{~h}$. Cortex Cinnamomi was added last followed by decocting for $20 \mathrm{~min}$. The final drug concentration was $1 \mathrm{~g} / \mathrm{mL}$ after being concentrated by heating.

2.2. Animal Experiments and Groups. The animal procedures and care in this study were approved by the Animal Ethics Committee of North China University of Science and Technology (approval number 2016016). Six-to-eight-weekold female Sprague Dawley (SD) rats were purchased from the Laboratory Animal Center of the Academy of Military Medical Sciences. All rats were housed in a room $\left(22^{\circ} \mathrm{C} \pm 2^{\circ} \mathrm{C}\right)$ with a 12 -hour light/dark cycle (7:00 AM to 7:00 $\mathrm{PM}$ ), with ad libitum access to water and food (fundamental diet, Beijing Vital River Laboratory Animal Technology Co., Ltd) in groups of 4 animals per cage. Vaginal smears were taken daily from all rats after conventional adaptive feeding for 1 week. A total of 38 rats with normal estrous cycles were selected. Eight rats were randomly selected as the normal group, and the remainder were induced to develop endometriosis by autologously transplanting uterine tissue fragments onto the peritoneal wall. In brief, the remaining 30 rats were administered $0.2 \mathrm{mg}$ of estradiol valerate $24 \mathrm{~h}$ before the surgery. Then, a $1.5-2 \mathrm{~cm}$ ventral midline incision was made after anesthetization induced by $2 \%$ pentobarbital sodium. The right side of the uterus was ligated and cut into $5 \times 5 \mathrm{~mm}$ squares after rinsing in sterile phosphate-buffered saline (PBS). Each tissue square was sutured to the left peritoneal wall, where blood vessels are abundant, using a single nonabsorbable 5-0 polypropylene suture. After 4 weeks, rats in diestrus were selected for a second surgery to assess the implants. The transplanted endometrium was manifest as a sac-like shape or transparent nodule with fluid accumulation, which was regarded as successful induction [19]. The lesion sizes were measured using digital millimeter calipers.

Twenty-four rats with endometriotic lesions were randomized into three groups. The blank and model groups were treated with normal saline, the letrozole group with letrozole, and the SFZY group with SFZYD. Letrozole was given every day at a dose of $0.2 \mathrm{mg} / \mathrm{kg} / \mathrm{d}$. In the clinical practice of Chinese herbal medicine, the prescription dose of SFZY is usually $46.5 \mathrm{~g}$ of herbal materials per day. The dose of SFZYD given to rats was determined according to a formula that translates doses between different species based on the animal's body surface area. The administered dose of rats $(\mathrm{g} / \mathrm{kg})=\left(46.5 \times 0.9 \times \sqrt[3]{(x / 60)^{2}}\right) / x(x$, the weight of rats; 60 , the weight of adult females). Generally, the rats that weighed $300 \mathrm{~g}$ were given $1.2 \mathrm{~g}$ of SFZYD daily. Normal saline was administered at a dose of $1 \mathrm{~mL} / \mathrm{d}$ in the blank and model groups. After 4 weeks of treatment, rats in diestrus were selected for their third surgery under anesthesia. Before anesthetization, fecal pellets were collected and immediately frozen in liquid nitrogen and then transferred to $-80^{\circ} \mathrm{C}$. The implants were measured with a digital millimeter caliper. Implant volume was calculated with the following formula: 
$V\left(\mathrm{~mm}^{3}\right)=0.52 \times$ length $\times$ width $\times$ height [19]. The implants were excised and fixed in $4 \%$ paraformaldehyde for immunohistochemistry.

2.3. Immunohistochemical Staining. After dewaxing, we placed the sections in $0.01 \mathrm{M}$ citrate solution to retrieve the antigen at high temperature and pressure for $30 \mathrm{~s}$ followed by cooling to room temperature. Then, the sections were incubated with $3 \%$ hydrogen peroxide solution at room temperature for $20 \mathrm{~min}$ to interrupt peroxidase activity; we incubated them with goat serum at room temperature for $30 \mathrm{~min}$ for nonspecific blocking. Subsequently, we added primary antibody anti-cyclooxygenase- $2(1: 200)$ to the sections and incubated them at $4^{\circ} \mathrm{C}$ overnight. Then, the secondary antibody (PV-9000, Zhongshan BioTech Co., Ltd., Beijing, China) was added, and sections were incubated at $37^{\circ} \mathrm{C}$ for $30 \mathrm{~min}$, prior to diaminobenzidine (DAB) staining. We used an Olympus BX53 microscope (Japan) to obtain images, and Image-Pro Plus6 (Media Cybernetics, Inc., Rockville, MD, USA) was used to analyze the mean optical density (MOD) of each image.

\subsection{Fecal DNA Extraction and Illumina MiSeq Sequencing.} Genomic DNA was extracted from every fecal sample, and the purity and concentration of DNA were determined by agarose gel electrophoresis. Then, the sample was diluted to the concentration of $1 \mathrm{ng} / \mu \mathrm{l}$ with sterile water, and the V3 and V4 regions of the 16s rDNA sequences were amplified using specific primers: forward primer-341F (5'CCTAYGGGRBGCASCAG- $3^{\prime}$ ) and reverse primer-806R (5'-GGACTACNNGGGTATCTAAT- $\left.3^{\prime}\right)$. PCR reactions were performed using the following thermal profile: $1 \mathrm{~min}$ at $98^{\circ} \mathrm{C}$ for initial denaturation, 30 cycles of $10 \mathrm{~s}$ at $98^{\circ} \mathrm{C}$ for denaturation, $30 \mathrm{~s}$ at $50^{\circ} \mathrm{C}$ for annealing, and $30 \mathrm{~s}$ at $72^{\circ} \mathrm{C}$ for elongation followed by a final elongation step for $5 \mathrm{~min}$ at $72^{\circ} \mathrm{C}$. We electrophoresed the samples to detect the PCR products using agarose gel as follows. The samples were mixed according to the concentration of PCR products, electrophoresis was performed by $1 \times$ TAE in $2 \%$ agarose gel to purify the PCR products, and the target bands were recycled. A library was constructed following the manufacturer's instructions for the Ion Plus Fragment Library Kit and sequenced with an Ion S5TMXL sequencer.

2.5. Bioinformatics Analysis. Cutadapt (V1.9.1, http://cutadapt. readthedocs.io/en/stable/) was used for quality filtering to retrieve the raw reads [20]. We analyzed the raw reads using VSEARCH (https://github.com/torognes/vsearch/) to remove chimeric sequences and acquired the clean reads [21]. Sequences were clustered into operational taxonomic units (OTUs) at a similarity of $97 \%$ by Uparse software (Uparse v7.0.1001, http://www.drive5.com/uparse/) [22]. We conducted matching of OTUs to bacteria (the threshold value was 0.8-1 [23]) using the Mothur algorithm and SILVA132 reference database. Taxonomic information was obtained, and the species composition was classified at different levels, such as kingdom, phylum, class, order, family, genus, and species. We performed fast multisequence alignment using MUSCLE software (version 3.8.31, http://www.drive5.com/muscle/) to obtain the phylogenetic relationships of all OTU sequences [24]). A species accumulation boxplot was generated with $\mathrm{R}$ software (version 2.15.3), and $\alpha$-diversity and $\beta$-diversity were generated by QIIME (version 1.9.1). ACE, Chao1, observed-species, Shannon, Simpson, and PD-whole tree indices were then used to assess the richness and evenness of gut microbiota followed by a post hoc two-sided Wilcoxon-Mann-Whitney test for determining the statistical dependence among groups. The unweighted and weighted UniFrac approach was used to measure the $\beta$-diversity among these four groups, and principal coordinate analysis (PCoA) was performed with $\mathrm{R}$ software (version 2.15.3) based on unweighted and weighted UniFrac data. AMOVA function was calculated using Mothur based on unweighted and weighted UniFrac algorithms to detect whether the species composition differences among groups were significant. We used the linear discriminant analysis (LDA) effect size (LEfSe) method to analyze the differences among groups at the phylum, class, order, family, and genus levels.

2.6. Statistical Analysis. Body weight gain and size and protein expression of endometriotic lesions were statistically analyzed using SPSS 23.0. Values are presented as means \pm standard deviation (SD). All data were first assessed for a normal distribution and for homogeneity of variance. Size and protein expression of endometriotic lesions were compared using a paired-sample $t$-test or one-way analysis of variance (ANOVA) with Tukey's post hoc tests. Body weight gain repeated over time was compared using two-way ANOVA with post hoc Student's $t$-tests. $P<0.05$ was considered statistically significant.

\section{Results}

3.1. Effects of Letrozole and SFZY on Endometriotic Lesion Growth and Weight Gain in Rats. As shown in Table 1, after treatment, the mean volume of endometriotic lesions decreased significantly in the letrozole group from $28.37 \pm 18.49$ to $3.57 \pm 4.49 \mathrm{~mm}^{3}(P<0.01)$ and in the SFZY group from $27.33 \pm 15.24$ to $8.05 \pm 7.82 \mathrm{~mm}^{3}(P<0.01)$; however, the mean volume of endometriotic implants in the model group increased significantly from $29.90 \pm 13.34$ to $45.26 \pm 20.74 \mathrm{~mm}^{3}(P<0.05)$. There was no difference between the letrozole and SFZY groups in lesion volume after medication administration $(P>0.05)$. As shown in Figure 1, the body weight gain in the letrozole group increased significantly compared with that in other groups $(P<0.05)$ from the second to the fourth weeks during treatment; there was no difference among the blank, model, or SFZY groups $(P>0.05)$.

3.2. Immunohistochemical Expression of Cyclooxygenase-2 in Endometriotic Lesions and Endometrium. According to immunohistochemical staining results (Figure 2), cyclooxygenase-2 (COX-2) protein expression in endometriotic 
TABle 1: Comparison of mean endometriotic implant volumes in the groups $(n=8)$.

\begin{tabular}{lcc}
\hline Group & Before medication $\left(\mathrm{mm}^{3}\right)$ & After medication $\left(\mathrm{mm}^{3}\right)$ \\
\hline Model & $29.90 \pm 13.34$ & $45.26 \pm 20.74^{*}$ \\
Letrozole & $28.37 \pm 18.49$ & $3.57 \pm 4.49^{* *}$ \\
SFZY & $27.33 \pm 15.24$ & $8.05 \pm 7.82^{\Delta^{* *}}$ \\
\hline
\end{tabular}

Note. ${ }^{\Delta} P<0.01$ compared with the model group after medication, ${ }^{*} P<0.05$ compared with the same group before medication, and ${ }^{* *} P<0.01$ compared with the same group before medication.

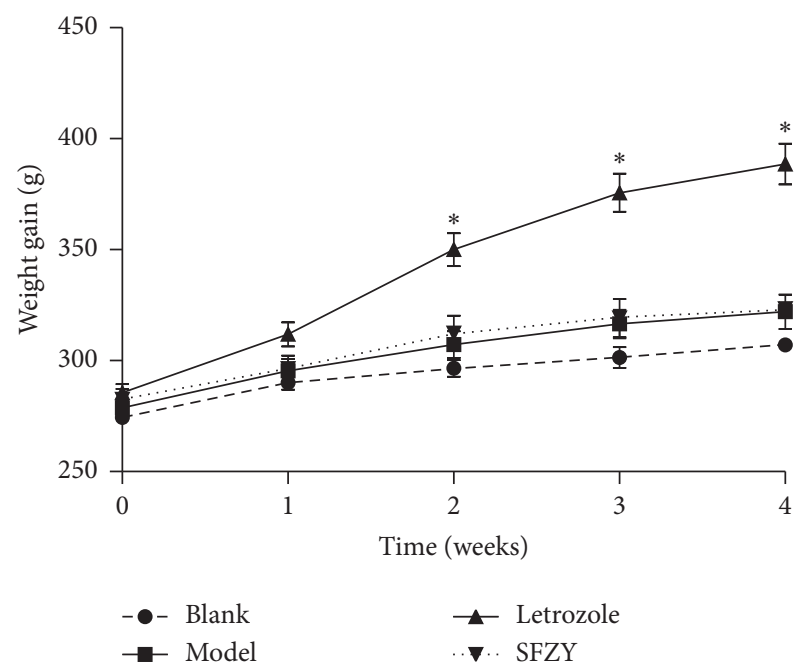

FIGURE 1: Body weight gain of rats in each group (two-way ANOVA with post hoc Student's $t$-tests, ${ }^{*} P<0.05$ ).

lesions of the letrozole and SFZY groups was significantly lower than that of the model group $(P<0.01)$. COX-2 protein expression in the endometrium of the model group was significantly higher than that of the blank group $(P<0.01)$, and expression in endometriotic lesions of the letrozole and SFZY groups was significantly lower than that of the model group $(P<0.01)$.

\subsection{Letrozole and SFZY Modulate the Composition of Gut} Microbiota in Endometriotic Rats. To detect the effects of letrozole and SFZY on gut microbial composition, we sequenced the 16S rRNA gene from DNA isolated from fecal samples from the four groups. An average of 85,049 reads were measured per sample, and 79,452 valid reads were obtained on average according to our quality blank measures. The effective rate of the quality blank was $93.49 \%$, and sequences were clustered into OTUs within $97 \%$ sequence identity. We obtained a total of 1070 OTUs from 24 samples (Figure 3(a)).

We used the $\alpha$-diversity to evaluate the richness and evenness of gut microbiota and ACE, Chaol, and observedspecies indices to evaluate the richness of the gut microbiota. As shown in Figure 3(b), the ACE index in the model group was lower than that in the blank group $(P<0.05)$, which indicated that the richness in the model group was significantly lower than that in the blank. We observed no statistical difference for the Chao 1 and observed-species indices between the blank and model groups. ACE, Chaol, and observed-species indices in the SFZY group that were similar to the blank group $(P>0.05)$ were significantly higher than those in the model and letrozole groups $(P<0.05)$. When we used Shannon, Simpson, and PD-whole tree indices to evaluate the richness and evenness of gut microbiota, the Simpson index in the model group was lower than that in the blank group (Figure 3(c), $P<0.05$ ), which indicated that the richness and evenness in the model group were lower. No statistical difference was observed for the Shannon and PDwhole tree indices between the blank and model groups; the Shannon, Simpson, and PD-whole tree indices in the SFZY group were also similar to the blank $(P>0.05)$, but significantly higher than those in the model group $(P<0.01)$. These results demonstrated that the species richness and evenness of the model group were significantly lower than those of the blank group and like those of the letrozole group. The effects of STZY might therefore have improved species richness and evenness that were impaired by endometriosis.

To detect the relationship between microbiota composition and specific taxa, we profiled the phyla across samples in each group. As shown in Figure 4(a), fecal samples from model rats contained a higher abundance of Firmicutes and lower abundance of Bacteroidetes and Proteobacteria than samples from blank rats. Fecal samples from letrozole and SFZY groups contained a lower abundance of Firmicutes and higher abundance of Bacteroidetes than the model group. Fecal samples from letrozole rats contained a lower abundance of Proteobacteria than samples from the other three groups. We confirmed these findings by analyzing the 10 most-abundant OTUs at the phylum level (Figure 4(b)). At the class level, fecal samples from model rats contained a higher abundance of Bacilli and lower abundance of Clostridia and Bacteroidia than the blank rats. In contrast, fecal samples from letrozole rats contained lower abundances of Bacilli and Clostridia and a higher abundance of Bacteroidia than model rats. Fecal samples from SFZY rats also contained a lower abundance of Bacilli and higher abundance of Bacteroidia and Clostridia than samples from model rats (Figure 4(c)). At the family level, fecal samples from model and letrozole rats contained a higher abundance of Lactobacillaceae and lower abundances of Ruminococcaceae and Peptostreptococcaceae than from blank and SFZY rats (Figure 4(d)). At the genus level, fecal samples from model and letrozole rats contained a higher abundance of Lactobacillus than from either blank or SFZY rats (Figure 4(e)).

To confirm the species composition differences among different groups, we profiled the $\beta$-diversity based on a species-abundance profiling table. Principal coordinate analysis (PCoA) was performed based on unweighted and weighted UniFrac algorithms (Figures 5(a) and 5(b)), and we calculated the AMOVA function using Mothur based on these unweighted and weighted UniFrac algorithms to detect whether the species composition differences among groups were significant. According to AMOVA function analysis, the species composition from each group was significantly different $(P<0.01)$. To further identify specific species in each group, we performed LDA effect size (LEfSe). As shown 

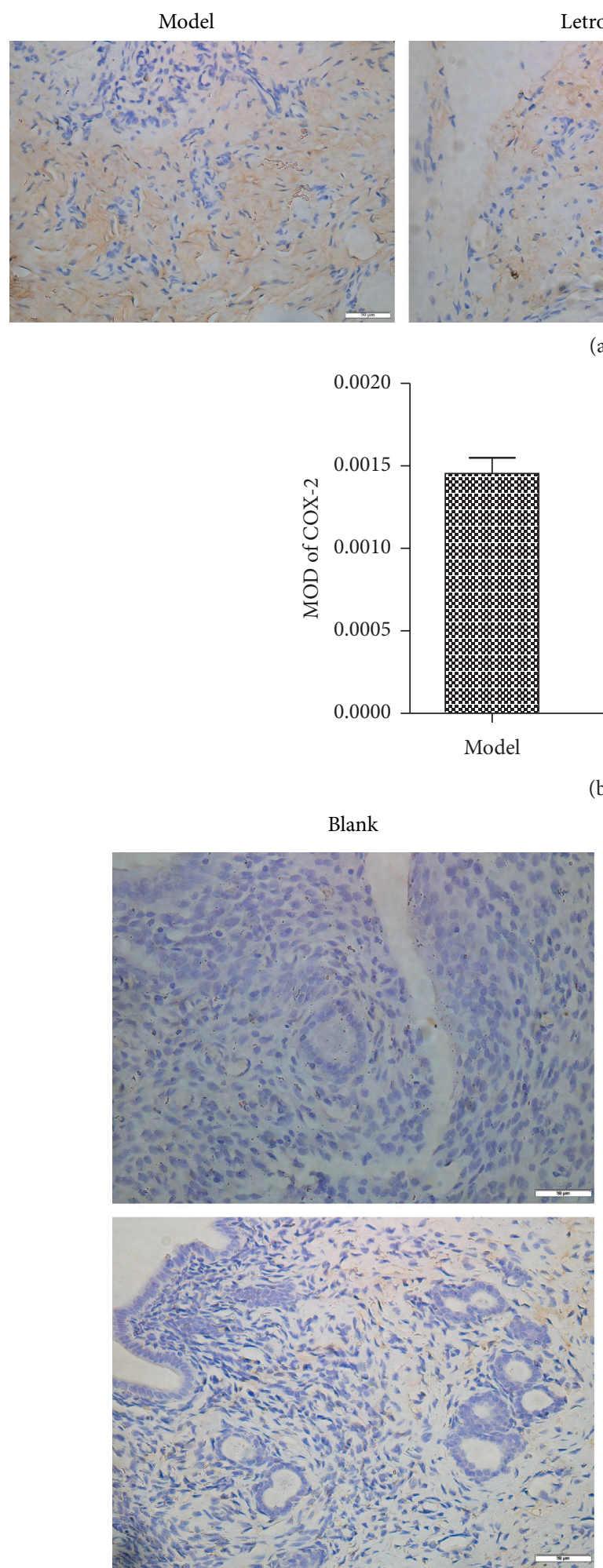

Letrozole

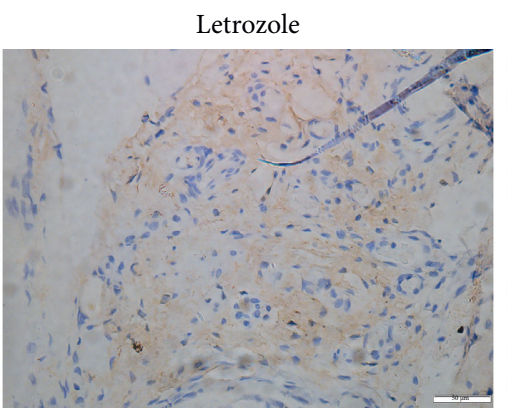

(a)

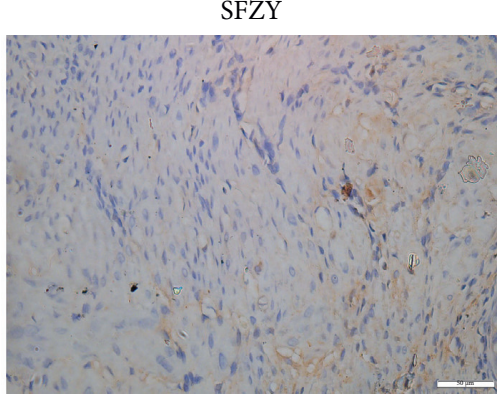

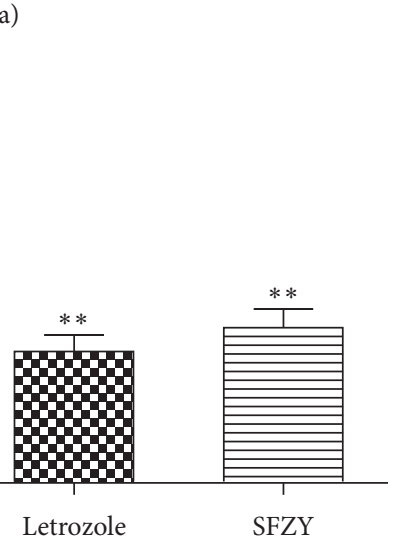

(b)
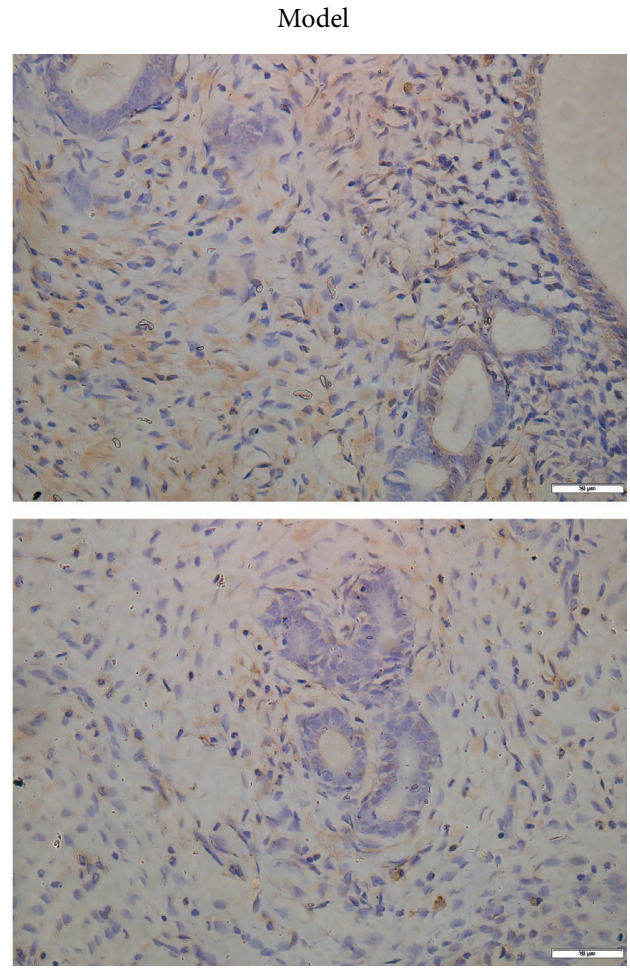

SFZY

(c)

Figure 2: Continued. 


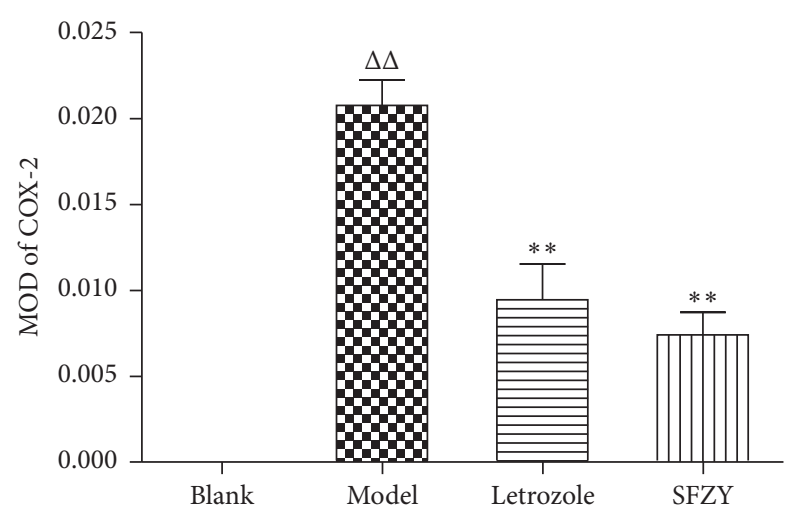

(d)

FIgURE 2: Representative images of immunohistochemical staining of COX-2 in endometriotic lesions and normal endometrium. (a) Sections stained for COX-2 in endometriotic lesions (magnification, $\times 400$ ). (b) Mean optical density (MOD) values of the COX-2 expression $(n=6)$; one-way ANOVA with Tukey's test, ${ }^{* *} P<0.01$ compared with the model group. (c) Sections stained for COX-2 in the endometrium (magnification, $\times 400)$. (d) Mean optical density (MOD) values of the COX-2 expression $(n=6)$; one-way ANOVA with Tukey's test, ${ }^{\Delta \Delta} P<0.01$ compared with the blank group and ${ }^{* *} P<0.01$ compared with the model group.

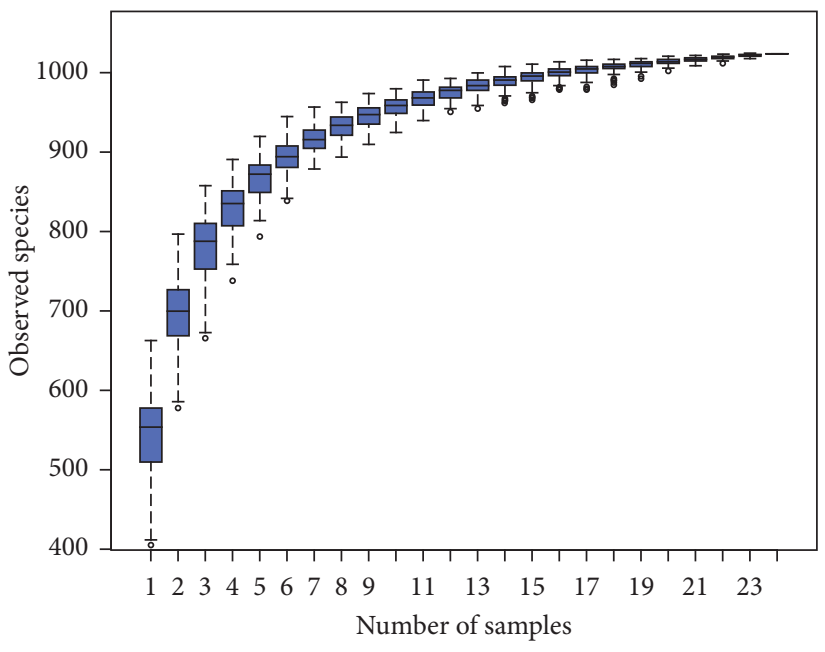

(a)

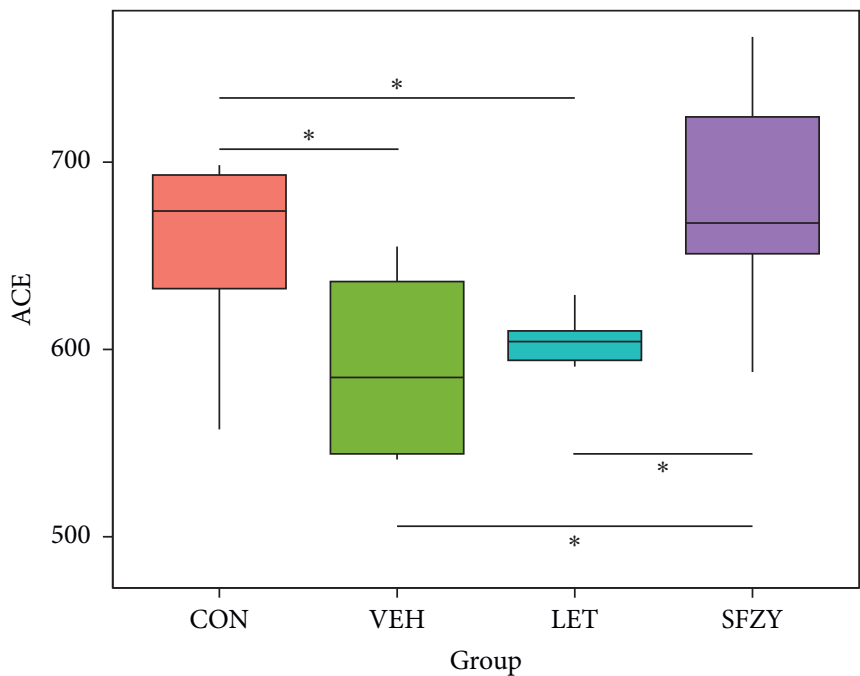

(b)

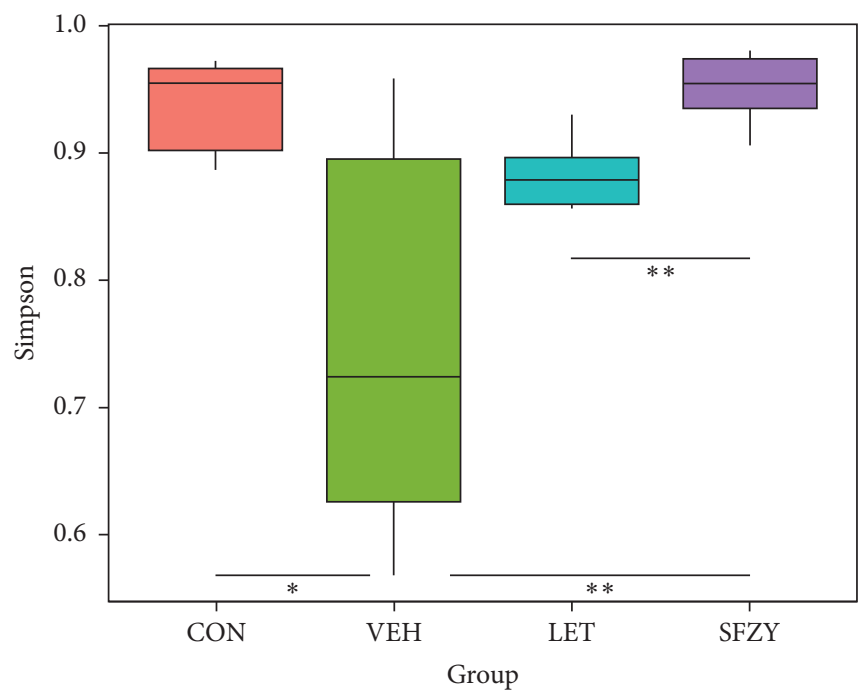

(c)

FIGURE 3: Gut microbiota composition is analyzed by $\alpha$-diversity $(n=6)$. (a) A species accumulation boxplot is used to evaluate species richness and whether the sample size is sufficient. (b, c) ACE and Simpson analyses are used to evaluate community richness and diversity within each group (Kruskal-Wallis test, a nonparametric test is used; ${ }^{*} P<0.05$ and ${ }^{* *} P<0.01$ ). 


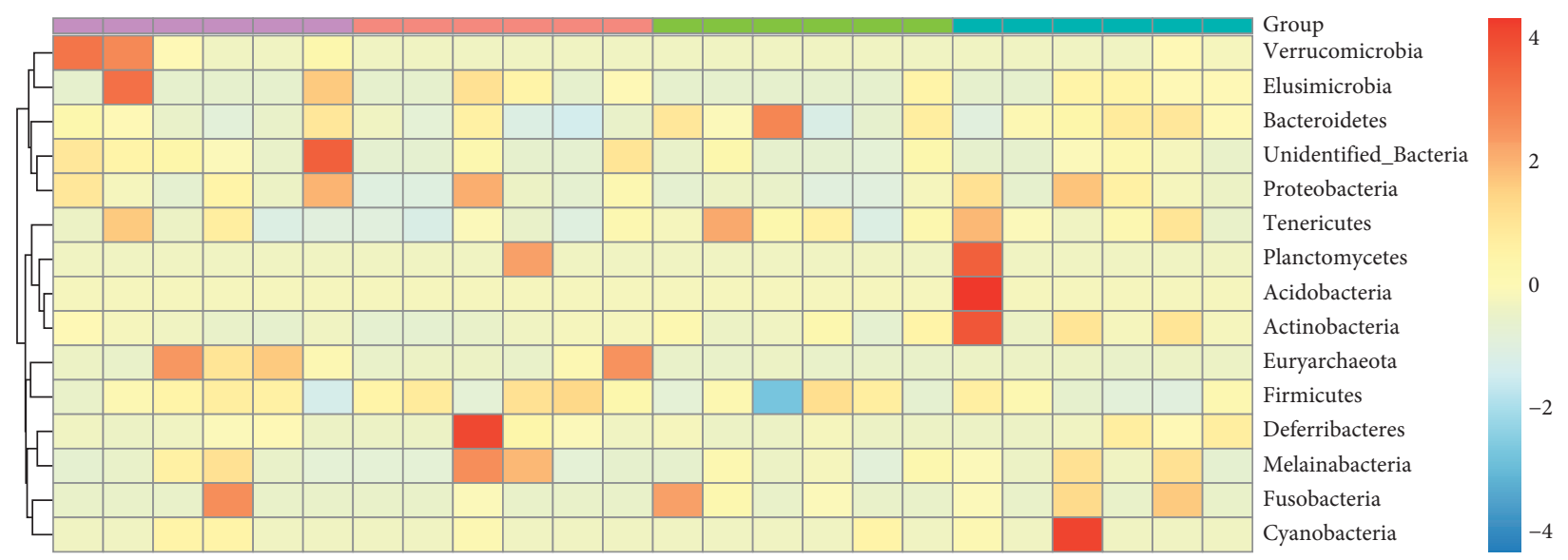

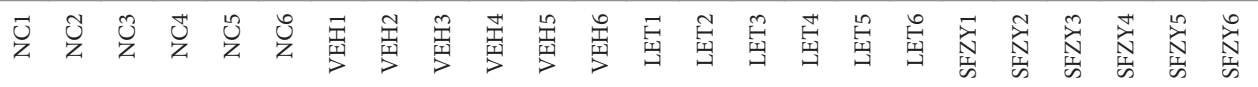

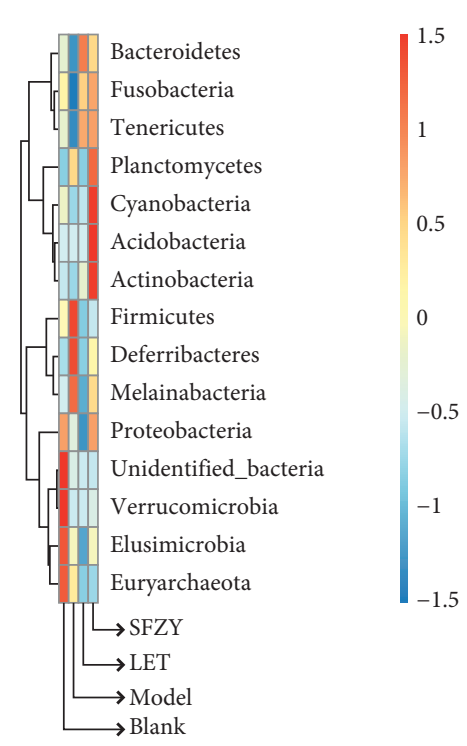

(a)
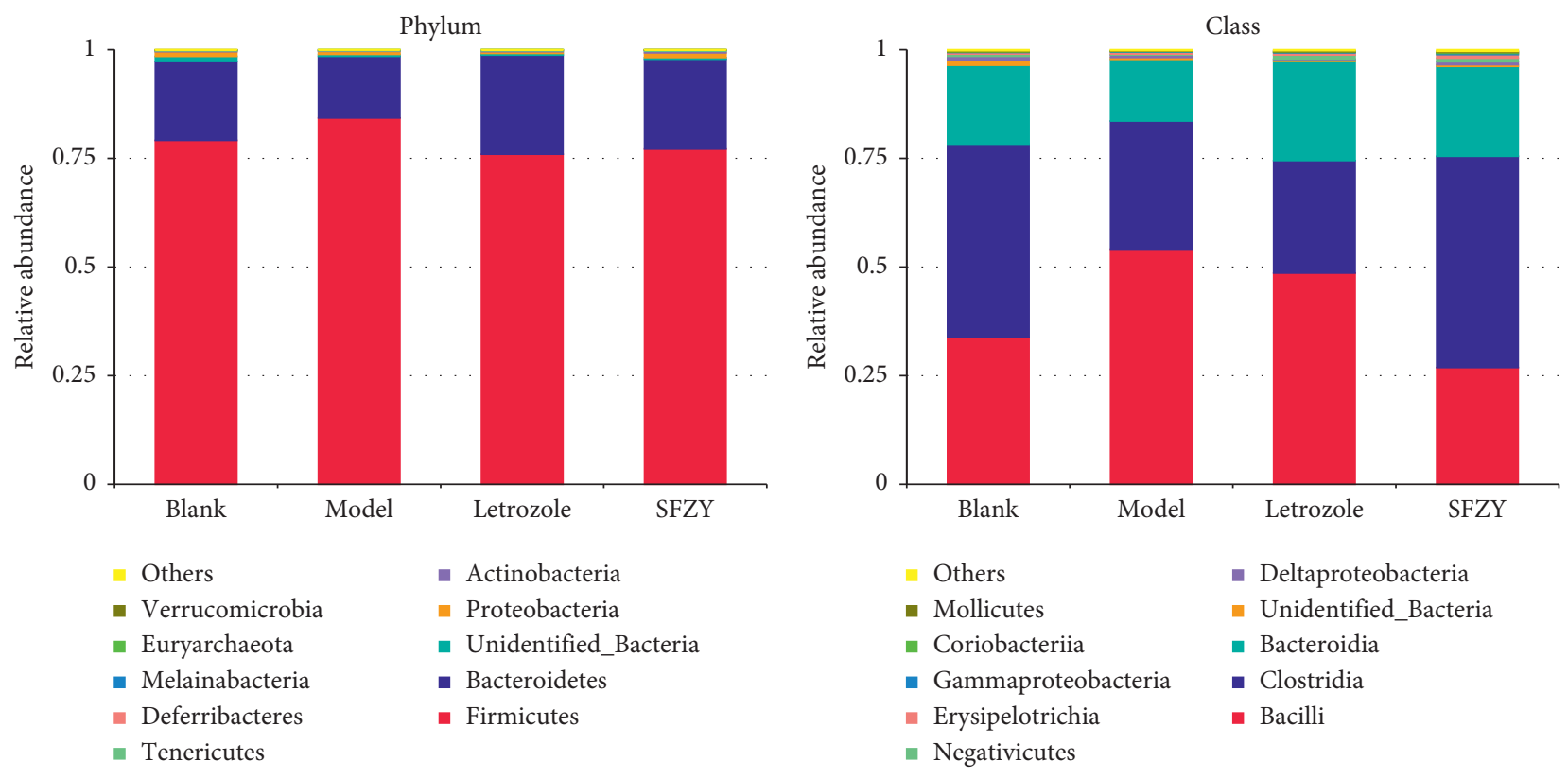

(b) 


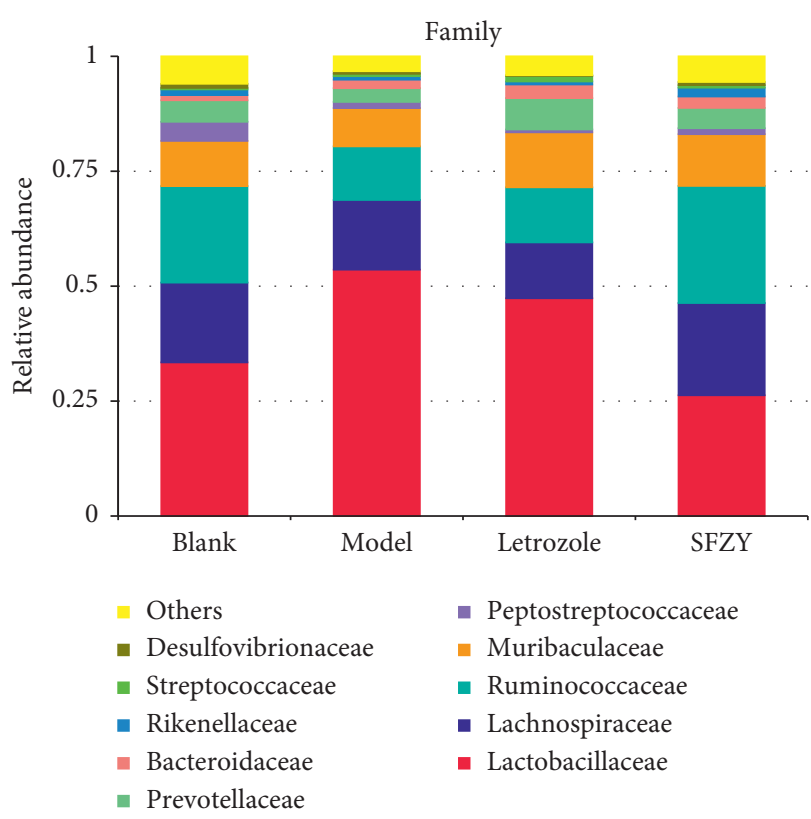

(d)

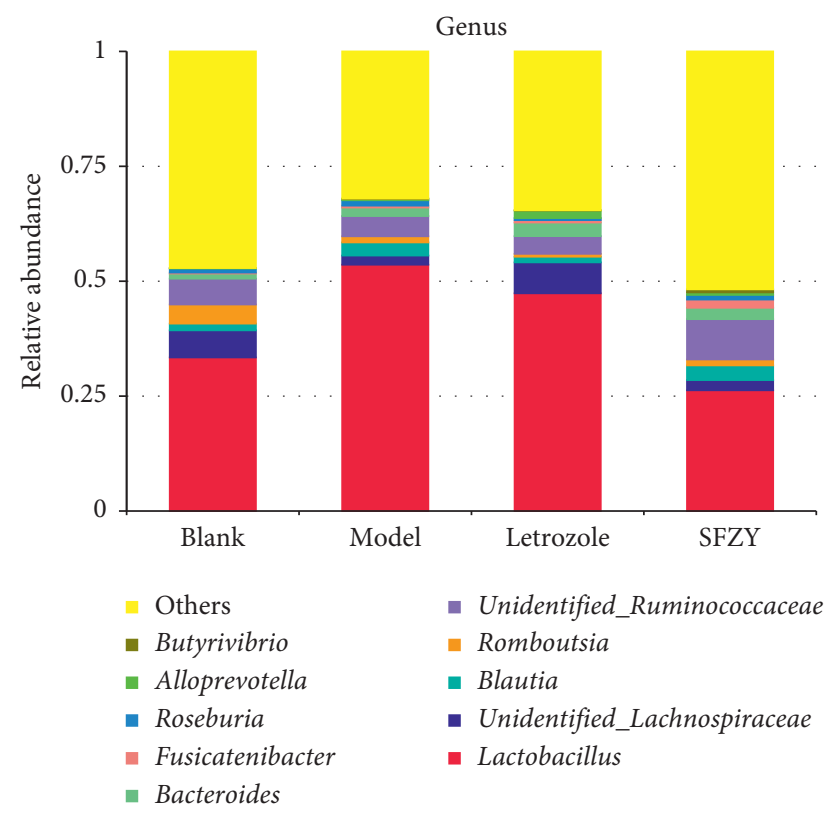

(e)

FIGURE 4: Analysis of species composition of gut microbiota among groups using OUT analysis $(n=6)$. (a) Heatmap representation of relative abundances of the phyla in fecal samples from each group. Microbiota distribution at the phylum level (b), class level (c), family level (d), and genus level (e).

in Figures 5(c) and 5(d), the histogram of LDA scores and cladogram indicated that the relative abundances of Lactobacillaceae and Beijerinckiaceae were enriched in model rats, and the relative abundance of Ruminococcaceae was higher in blank rats than in model rats at the family level. As shown in Figures 5(e) and 5(f), the histogram of LDA scores and cladogram indicated that the relative abundance of Ruminococcaceae was higher in blank rats than in model and letrozole rats at the family level. As shown in Figures 5(g) and 5(h), the histogram of LDA scores and cladogram indicated that the relative abundance of Ruminococcaceae was higher in SFZY rats than in model and blank rats at the family level, and the relative abundance of Lactobacillus was higher in model rats than in SFZY and blank rats at the genus level.

\section{Discussion}

There are nearly $10^{14}$ microbes in the human body, approximately 10 times the number of human cells, and the impact of the gut microbiota on human health and disease has attracted increasing scientific interest [25-28] in recent years, including in areas such as obesity, inflammatory diseases, cardiovascular diseases, and tumors. The gut microbiota is believed to be involved in the pathologic process of EMs [29]. Existing experimental studies primarily focus on mouse models of endometriosis. For example, Yuan et al. demonstrated that the Firmicutes/Bacteroidetes ratio was elevated in mice with endometriosis, as were the abundances of Firmicutes and Bacteroidetes in the gut microbiota flora of mice 42 days after modeled induction [15]; this is consistent with our study. In contrast, Josefine et al. investigated the gut microbiota of mice 21 days after inducing endometriosis and found that there was no effect on the microbiota [30]. Our contrasting conclusions may be due to the difference in sampling times. Most investigators posit that, under conditions of high fat, the gut microbiota of rodents increases the abundance of Firmicutes and decreases the abundance of Bacteroidetes. The increased Firmicutes/ Bacteroidetes ratio in the gut appears to weaken the barrier function of the intestinal epithelium and increase LPS levels in plasma, which is conducive to the formation of endotoxemia [31]. The translocation of LPS from the intestinal epithelium to the pelvic cavity is then conducive to the maintenance of pelvic inflammation [13], which might provide a favorable environment for ectopic endometrial implantation. In addition, Sangappa et al.'s study suggested that the abundance of Firmicutes in the gut of mice with endometriosis decreased while that of Bacteroidetes increased [16], which contradicts the conclusions in our current study. As such, the specific role of gut microbiota in the development of endometriosis needs to be studied and elucidated further. We also found a decrease in the abundance of Ruminococcaceae bacteria in the model group. Interestingly, some studies have suggested that Ruminococcaceae is negatively correlated with apoptosis of intestinal epithelial cells and IL-6 levels in mice [32], indicating that decreased abundance of Ruminococcaceae may aggravate pelvic inflammation.

Our research results showed that both letrozole and SFZYD inhibited the progression of ectopic lesions in rats with endometriosis and reduced inflammatory response by inhibiting the expression of COX-2 in ectopic and eutopic endometrial tissues. After comparing the differences in gut 


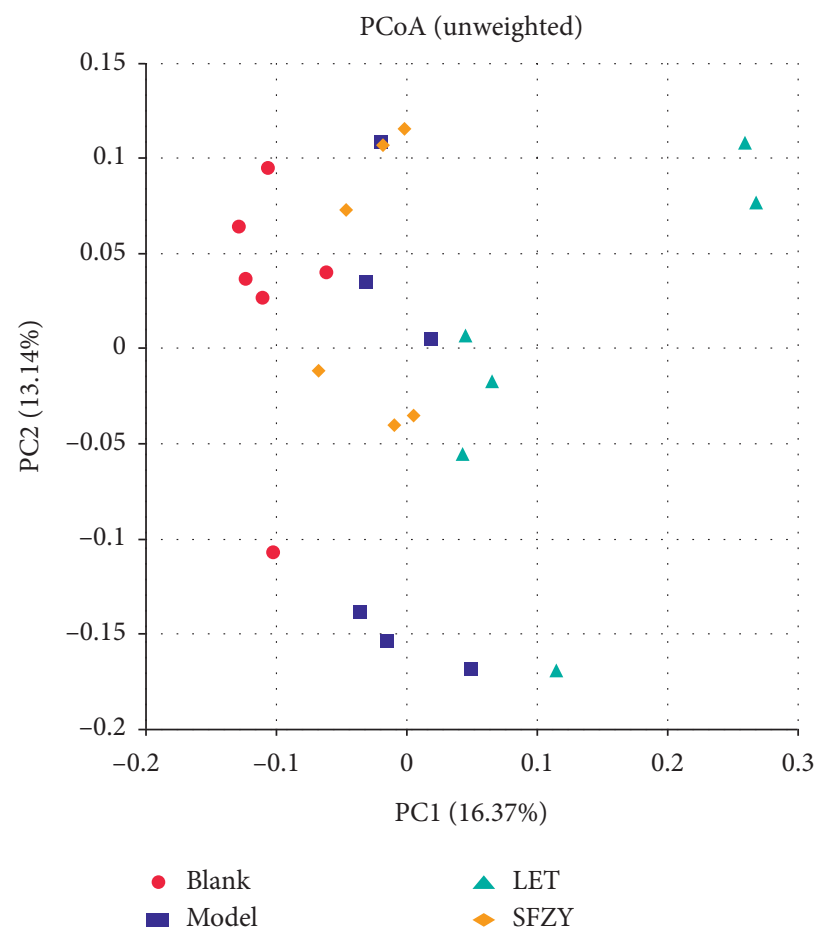

(a)

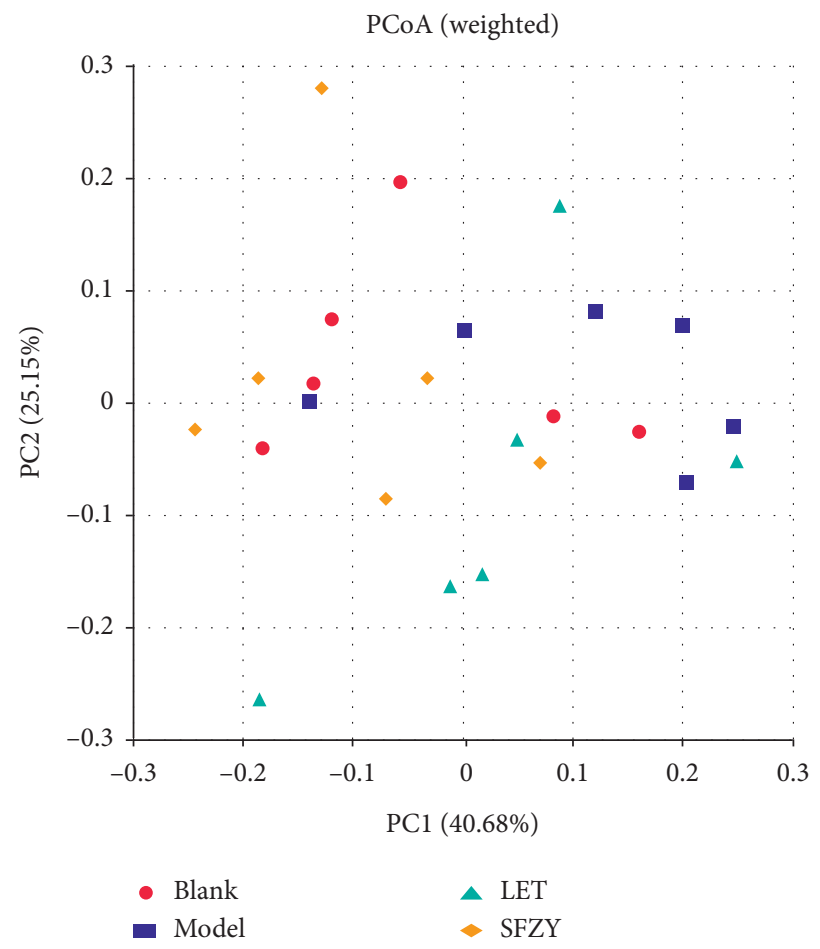

(b)

Figure 5: Continued. 


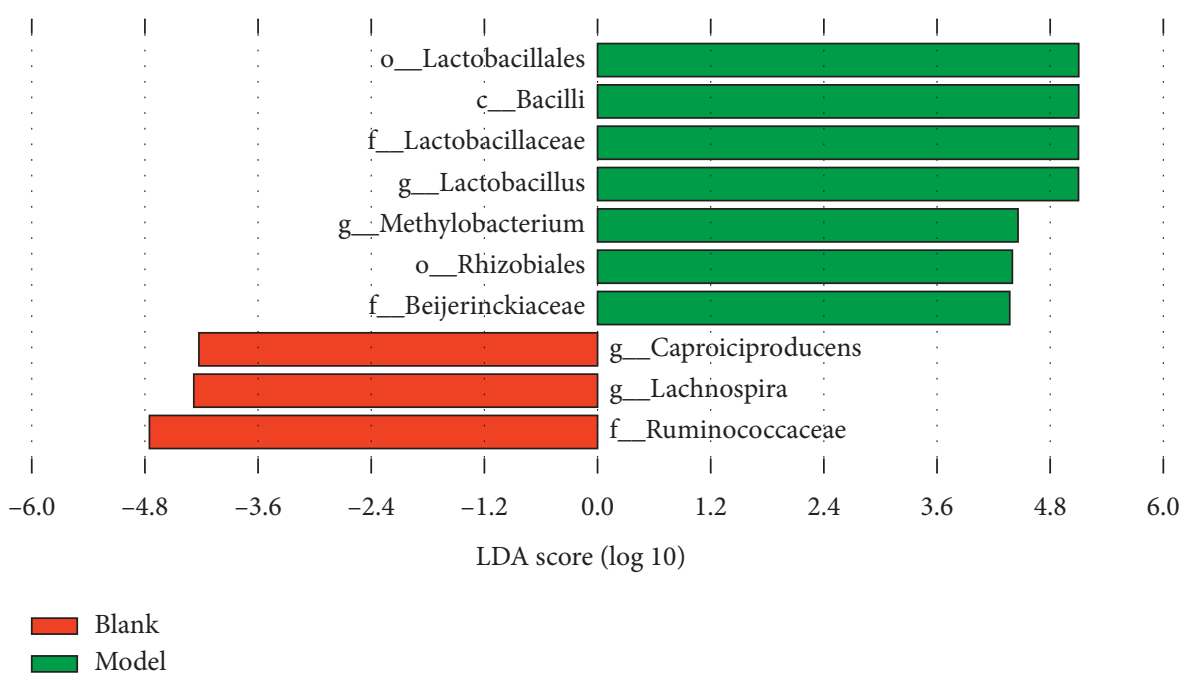

(c)

Cladogram

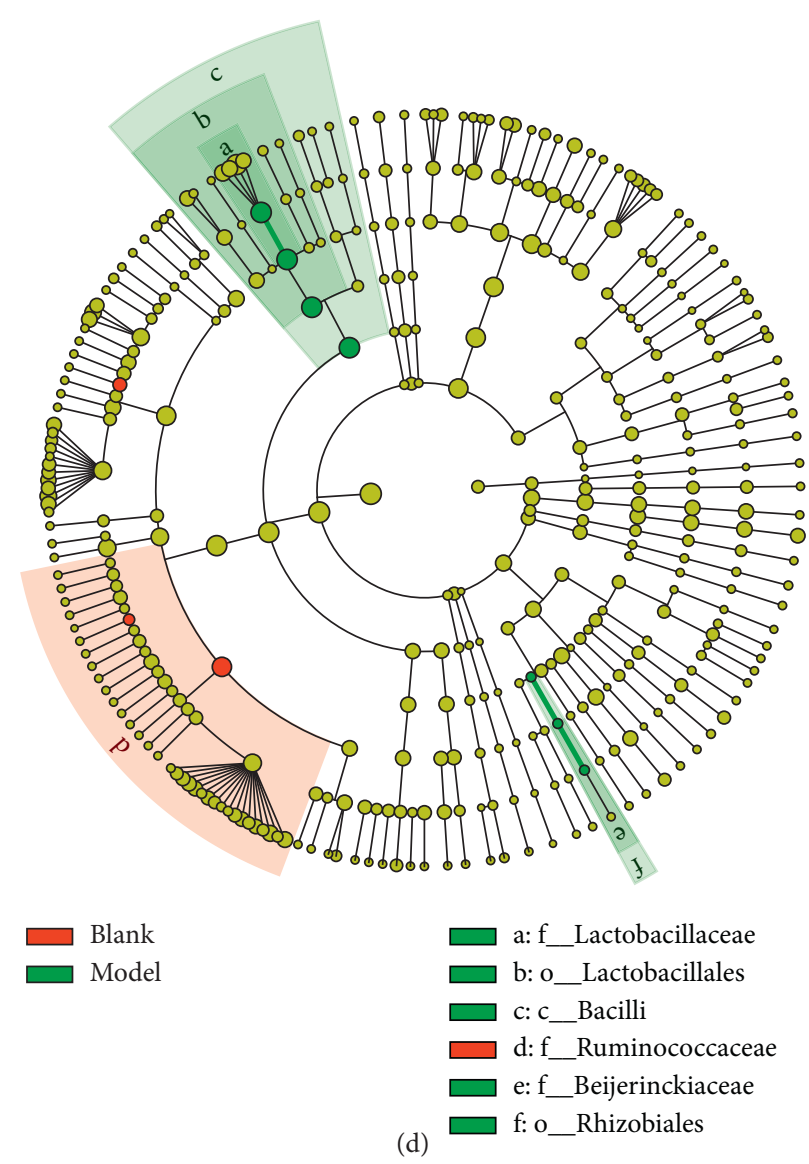

Figure 5: Continued. 


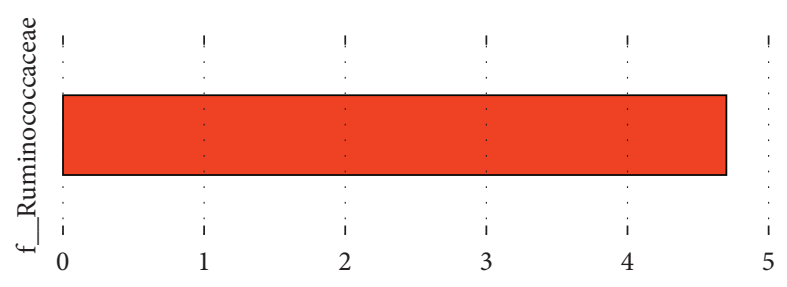

LDA score $(\log 10)$

$\square$ Blank

(e)

Cladogram

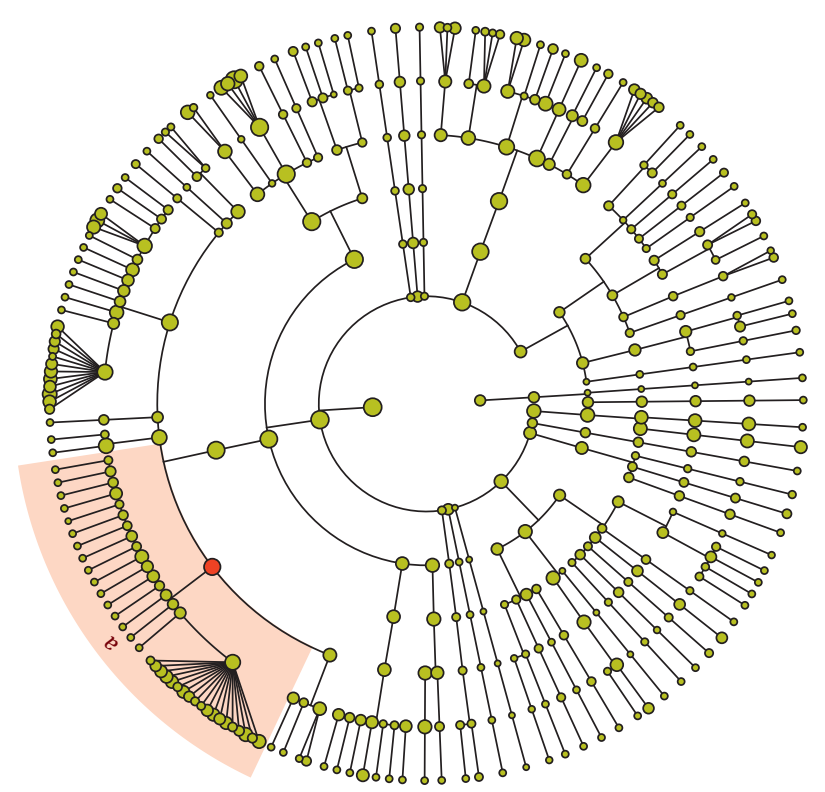

$\square$ Blank

(f)

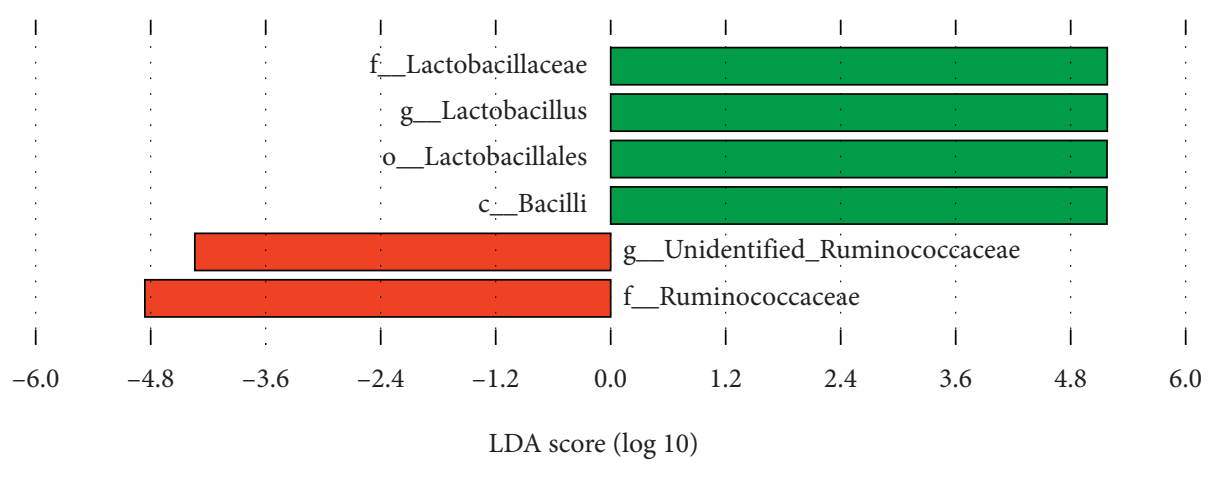

$\square$ SFZY

Model

(g)

Figure 5: Continued. 


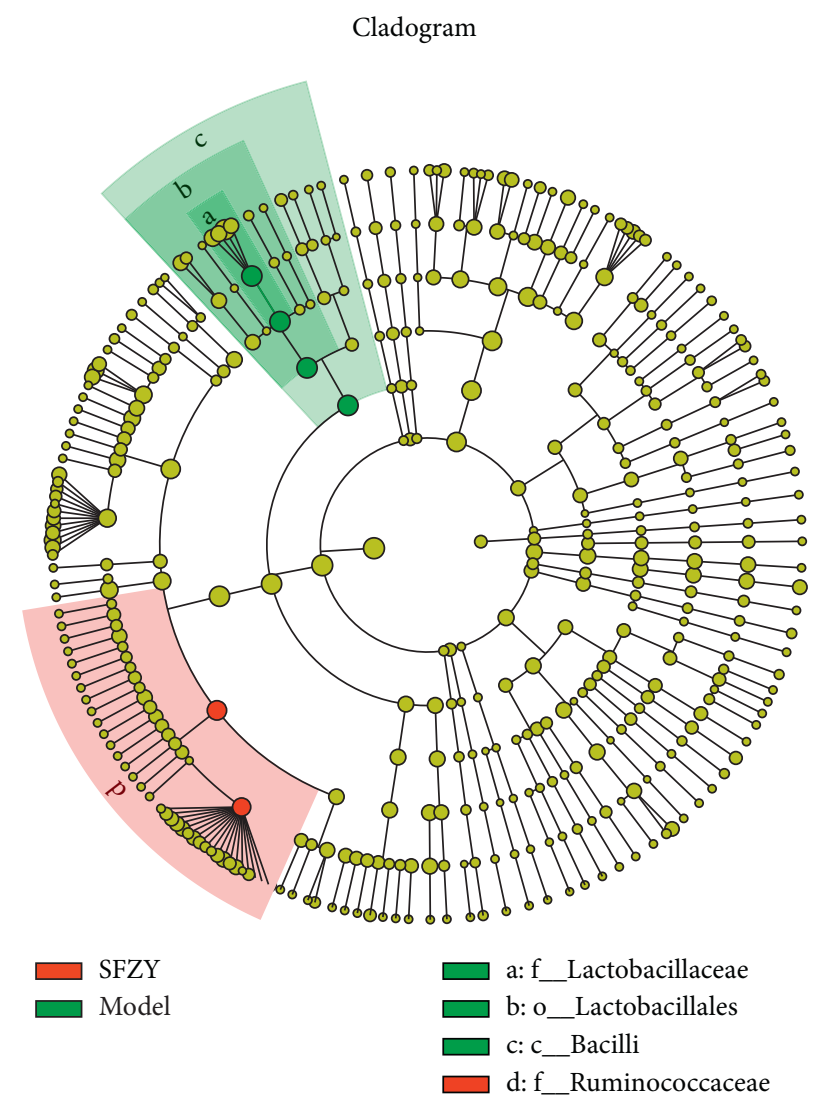

(h)

Figure 5: Comparison of gut microbiota among groups using PCoA and LEfSe analyses. (a, b) PCoA analysis based on unweighted and weighted UniFrac algorithms among groups. (c, d) Histogram of LDA scores and cladogram for differentially abundant genera between blank and model groups. (e, f) Histogram of LDA scores and cladogram for differentially abundant genera among blank, model, and cc vcx letrozole groups. (g, h) Histogram of LDA scores and cladogram for differentially abundant genera among blank, model, and SFZY groups.

microbiota of the four groups of rats, we found that both medications reduce the Firmicutes/Bacteroidetes ratio, which might be one of the mechanisms underlying the reduction in the inflammatory response in rats with endometriosis. Based on previous studies, an increase in the Firmicutes/Bacteroidetes ratio is likely related to a low-grade inflammation that not only affects the gut but also affects other organs [33]. We therefore speculated that the reduction in the Firmicutes/ Bacteroidetes ratio by these two drugs may be beneficial in reducing inflammation induced by endometriosis. However, in the present study, we could not prove that the correlation between gut microbiota and inflammation was directly caused by endometriosis; this needs to be confirmed further by fecal-transplant experiments.

After treatment, estrous cyclicity in the letrozole group ceased, with the rats remaining in diestrus. To ensure the accuracy of our experimental conclusions, we sampled the other groups of rats on diestrus. In animal studies, long-term administration of letrozole has been found to lead to obesity and polycystic ovarian changes such that letrozole has been used to induce polycystic ovary syndrome in animal models $[34,35]$. In clinical trials, the major adverse reactions to letrozole in the treatment of endometriosis were related to the low concentrations of circulating estrogen, i.e., vaginal dryness, hot flashes, and osteoporosis; these reactions have limited letrozole's clinical application [25]. The effect of letrozole on gut microbiota is mostly found in studies involving animal models of polycystic ovarian syndrome (PCOS). Scott et al. showed that, in a letrozole-induced mouse model, the abundance of Firmicutes in the gut increased while that of Bacteroidetes decreased [36], which is inconsistent with the conclusions of the present study. The dosage of letrozole in Scott et al.'s study was, however, much higher than in our study, and this may be a reason for the difference in the conclusions drawn. Our analysis of the $\alpha$-diversity confirmed that letrozole impaired the richness and evenness of gut microbiota in rats and diminished the abundance of Ruminococcaceae. In addition, when we evaluated the changes in body weights of rats in each group, we demonstrated that the upregulation of the Firmicutes/ Bacteroidetes ratio was generally associated with obesity [25]. Although rat body weight in the letrozole group increased significantly, the Firmicutes/Bacteroidetes ratio in this group was not significantly different from the blank group. Our LEfSe comparison of specific taxa between the letrozole and blank groups showed that the increased 
abundance of Streptococcaceae in the gut of letrozole-treated rats was also related to rat obesity [37]. Therefore, the effect of letrozole on the gut microbiota of clinical patients needs to be further evaluated.

In the SFZY group, the abundance of Bacilli and Ruminococcaceae increased markedly at the class level. Based on the $\beta$-diversity indices, the comparison between the blank group and the SFZY group confirmed that there was no statistically significant difference between the two groups, suggesting that SFZYD normalized the gut microbiota in rats with endometriosis. This included reducing the Firmicutes/Bacteroidetes ratio and increasing the abundance of Ruminococcaceae, which could restore the impaired intestinal barrier function and indirectly alleviate the inflammatory response of rats with endometriosis as we have discussed above. It is worth noting that SFZYD has been shown to reduce inflammation and promote lipid metabolism in a high-fat diet mouse model [38]. In addition, the network pharmacology analysis showed that the lipid metabolism-related pathway constituted potential protein targets of SFZYD [39]. The abundance of Ruminococcaceae and Prevotellaceae was increased significantly in SFZY rats relative to model rats, and these probiotics decompose complex carbohydrates into short-chain fatty acids (SCFAs) that are energy sources for intestinal epithelial cells and protective factors in gut inflammation $[40,41]$. As a consequence, SFZY would likely restore impaired gut microbiota and then promote SCFA production to improve the gut barrier, which would play a beneficial role in endometriosis treatment by reducing inflammation in the ectopic endometrium and in the pelvic cavity.

\section{Conclusions}

In this study, we confirmed that modulation of gut microbiota exists in endometriotic rats and that it is principally characterized by an augmented Firmicutes/Bacteroidetes ratio and reduced abundance of Ruminococcaceae. We postulate that both letrozole and SFZYD reduced the inflammatory response of ectopic and eutopic endometrial tissues, which might be related to the decrease in the Firmicutes/Bacteroidetes ratio.

\section{Data Availability}

The data used to support the findings of this study are available upon request from the corresponding author and the first author.

\section{Conflicts of Interest}

The authors declare that there are no conflicts of interest in this study.

\section{Authors' Contributions}

Ying Cao analyzed the data and drafted the paper. Chunhua Jiang and Yongsen Jia carried out the main experiments. Dingjie Xu participated in the studies partly. Yanyan $\mathrm{Yu}$ designed the research protocol.

\section{Acknowledgments}

The authors thank LetPub for its linguistic assistance during the preparation of this manuscript. This study was supported by a grant from the National Natural Science Foundation of China (Grant no. 81603455).

\section{References}

[1] A. Harlev, S. Gupta, and A. Agarwal, "Targeting oxidative stress to treat endometriosis," Expert Opinion on Therapeutic Targets, vol. 19, no. 11, pp. 1447-1464, 2015.

[2] J. A. Sampson, "Peritoneal endometriosis due to the menstrual dissemination of endometrial tissue into the peritoneal cavity," American Journal of Obstetrics and Gynecology, vol. 14, no. 4, pp. 422-469, 1927.

[3] S. H. Ahn, A. K. Edwards, S. S. Singh, S. L. Young, B. A. Lessey, and C. Tayade, "IL-17A contributes to the pathogenesis of endometriosis by triggering proinflammatory cytokines and angiogenic growth factors," The Journal of Immunology, vol. 195, no. 6, pp. 2591-2600, 2015.

[4] S. H. Ahn, S. P. Monsanto, C. Miller, S. S. Singh, R. Thomas, and C. Tayade, "Pathophysiology and immune dysfunction in endometriosis," BioMed Research International, vol. 2015, Article ID 795976, 12 pages, 2015.

[5] W. Nothnick and Z. Alali, "Recent advances in the understanding of endometriosis: the role of inflammatory mediators in disease pathogenesis and treatment," F1000 Research, vol. 5, p. F1000, 2016.

[6] Y.-H. Lin, Y.-H. Chen, H.-Y. Chang, H.-K. Au, C.-R. Tzeng, and Y.-H. Huang, "Chronic niche inflammation in endometriosis-associated infertility: current understanding and future therapeutic strategies," International Journal of Molecular Sciences, vol. 19, no. 8, p. 2385, 2018.

[7] R. Gazvani and A. Templeton, "Peritoneal environment, cytokines and angiogenesis in the pathophysiology of endometriosis," Reproduction, vol. 123, no. 2, pp. 217-226, 2002.

[8] H. Rakhila, M. Al-Akoum, M.-E. Bergeron et al., "Promotion of angiogenesis and proliferation cytokines patterns in peritoneal fluid from women with endometriosis," Journal of Reproductive Immunology, vol. 116, pp. 1-6, 2016.

[9] R. Cosín, J. Gilabert-Estellés, L. A. Ramón et al., "Influence of peritoneal fluid on the expression of angiogenic and proteolytic factors in cultures of endometrial cells from women with endometriosis," Human Reproduction, vol. 25, no. 2, pp. 398-405, 2010.

[10] K. N. Khan, M. Kitajima, K. Hiraki et al., "Escherichia coli contamination of menstrual blood and effect of bacterial endotoxin on endometriosis," Fertility and Sterility, vol. 94, no. 7, pp. 2860-2863, 2010.

[11] D. Karmarkar and K. L. Rock, "Microbiota signalling through MyD88 is necessary for a systemic neutrophilic inflammatory response," Immunology, vol. 140, no. 4, pp. 483-492, 2013.

[12] R. Emani, C. Alam, S. Pekkala, S. Zafar, M. R. Emani, and A. Hänninen, "Peritoneal cavity is a route for gut-derived microbial signals to promote autoimmunity in non-obese diabetic mice," Scandinavian Journal of Immunology, vol. 81, no. 2, pp. 102-109, 2015.

[13] M. Schoeler and R. Caesar, "Dietary lipids, gut microbiota and lipid metabolism," Reviews in Endocrine and Metabolic Disorders, vol. 20, no. 4, pp. 461-472, 2019.

[14] R. Flores, J. Shi, B. Fuhrman et al., "Fecal microbial determinants of fecal and systemic estrogens and estrogen 
metabolites: a cross-sectional study," Journal of Translational Medicine, vol. 10, no. 1, p. 253, 2012.

[15] M. Yuan, D. Li, Z. Zhang, H. Sun, M. An, and G. Wang, "Endometriosis induces gut microbiota alterations in mice," Human Reproduction, vol. 33, no. 4, pp. 607-616, 2018.

[16] S. B. Chadchan, M. Cheng, L. A. Parnell et al., "Antibiotic therapy with metronidazole reduces endometriosis disease progression in mice: a potential role for gut microbiota," Human Reproduction, vol. 34, no. 6, pp. 1106-1116, 2019.

[17] Z. Li, H. Liu, Z. He, G. Zhang, and J. Lang, "Effects of cisplatin and letrozole on surgically induced endometriosis and comparison of the two medications in a rat model," European Journal of Pharmaceutical Sciences, vol. 93, pp. 132-140, 2016.

[18] G. Zhu, C. Jiang, X. Yan, S. Zhao, D. Xu, and Y. Cao, "Shaofu zhuyu decoction regresses endometriotic lesions in a rat model," Evidence-Based Complementary and Alternative Medicine, vol. 2018, Article ID 3927096, 7 pages, 2018.

[19] B. Yilmaz, M. Ozat, S. Kilic et al., "Atorvastatin causes regression of endometriotic implants in a rat model," Reproductive BioMedicine Online, vol. 20, no. 2, pp. 291-299, 2010.

[20] K. P. Aßhauer, B. Wemheuer, R. Daniel, and P. Meinicke, "Tax4Fun: predicting functional profiles from metagenomic 16S rRNA data: fig. 1," Bioinformatics, vol. 31, no. 17, pp. 2882-2884, 2015.

[21] T. Rognes, T. Flouri, B. Nichols, C. Quince, and F. Mahé, "VSEARCH: a versatile open source tool for metagenomics," PeerJ, vol. 4, p. e2584, 2016.

[22] B. J. Haas, D. Gevers, A. M. Earl et al., "Chimeric 16S rRNA sequence formation and detection in Sanger and 454-pyrosequenced PCR amplicons," Genome Research, vol. 21, no. 3, pp. 494-504, 2011.

[23] R. C. Edgar, "UPARSE: highly accurate OTU sequences from microbial amplicon reads," Nature Methods, vol. 10, no. 10, pp. 996-998, 2013.

[24] C. Quast, E. Pruesse, P. Yilmaz et al., "The SILVA ribosomal RNA gene database project: improved data processing and web-based tools," Nucleic Acids Research, vol. 41, pp. D590D596, 2013.

[25] M. Sharma, Y. Li, M. L. Stoll, and T. O. Tollefsbol, "The epigenetic connection between the gut microbiome in obesity and diabetes," Frontiers in Genetics, vol. 10, p. 1329, 2020.

[26] A. Fasano, "All disease begins in the (leaky) gut: role of zonulin-mediated gut permeability in the pathogenesis of some chronic inflammatory diseases," F1000 Research, vol. 9, p. F1000, 2020.

[27] H. Xu, X. Wang, W. Feng et al., "The gut microbiota and its interactions with cardiovascular disease," Microbial Biotechnology, vol. 13, no. 3, pp. 637-656, 2020.

[28] L. Shui, X. Yang, J. Li, C. Yi, Q. Sun, and H. Zhu, "Gut microbiome as a potential factor for modulating resistance to cancer immunotherapy," Frontiers in Immunology, vol. 10, p. 2989, 2020.

[29] M. W. Laschke and M. D. Menger, "The gut microbiota: a puppet master in the pathogenesis of endometriosis?" American Journal of Obstetrics and Gynecology, vol. 215, no. 1, pp. e1-68, 2016.

[30] J. Hantschel, S. Weis, K.-H. Schäfer et al., "Effect of endometriosis on the fecal bacteriota composition of mice during the acute phase of lesion formation," PLoS One, vol. 14, no. 12, Article ID e0226835, 2019.

[31] S. Gómez-Zorita, L. Aguirre, I. Milton-Laskibar et al., "Relationship between changes in microbiota and liver steatosis induced by high-fat feeding-a review of rodent models," Nutrients, vol. 11, no. 9, p. 2156, 2019.
[32] J. Meng, S. Banerjee, L. Zhang et al., "Opioids impair intestinal epithelial repair in HIV-infected humanized mice," Frontiers in Immunology, vol. 10, p. 2999, 2020.

[33] A. Pascale, N. Marchesi, S. Govoni, A. Coppola, and C. Gazzaruso, "The role of gut microbiota in obesity, diabetes mellitus, and effect of metformin: new insights into old diseases," Current Opinion in Pharmacology, vol. 49, pp. 1-5, 2019.

[34] A. Nallathambi and R. Bhargavan, "Regulation of estrous cycle by Cynodon dactylon in letrozole induced polycystic ovarian syndrome in Wistars albino rats," Anatomy \& Cell Biology, vol. 52, no. 4, pp. 511-517, 2019.

[35] I. Ortega, A. Sokalska, J. A. Villanueva et al., "Letrozole increases ovarian growth and Cyp17al gene expression in the rat ovary," Fertility and Sterility, vol. 99, no. 3, pp. 889-896, 2013.

[36] S. T. Kelley, D. V. Skarra, A. J. Rivera, and V. G. Thackray, "The gut microbiome is altered in a letrozole-induced mouse model of polycystic ovary syndrome," PLoS One, vol. 11, no. 1, Article ID e0146509, 2016.

[37] M. E. Palomo-Buitrago, M. Sabater-Masdeu, J. M. MorenoNavarrete et al., "Glutamate interactions with obesity, insulin resistance, cognition and gut microbiota composition," Acta Diabetologica, vol. 56, no. 5, pp. 569-579, 2019.

[38] M. Hong, J. Jung, H. S. Park et al., "Shaofu zhuyu decoction ameliorates obesity-mediated hepatic steatosis and systemic inflammation by regulating metabolic pathways," PLoS One, vol. 12, no. 6, Article ID e0178514, 2017.

[39] P. Liu, J. A. Duan, G. Bai, and S. L. Su, "Network pharmacology study on major active compounds of siwu decoction analogous formulae for treating primary dysmenorrhea of gynecology blood stasis syndrome," Zhongguo Zhong Yao Za Zhi, vol. 39, no. 1, pp. 113-120, 2014.

[40] G. Wang, S. Sun, X. Wu et al., "Intestinal environmental disorders associate with the tissue damages induced by perfluorooctane sulfonate exposure," Ecotoxicology and Environmental Safety, vol. 197, Article ID 110590, 2020.

[41] S.-J. Yue, J. Liu, W.-X. Wang et al., "Berberine treatmentemergent mild diarrhea associated with gut microbiota dysbiosis," Biomedicine \& Pharmacotherapy, vol. 116, Article ID 109002, 2019. 